\title{
Hydrogen-Bonding Capability of a Templating Difluorotoluene Nucleotide Residue in an RB69 DNA Polymerase Ternary Complex
}

\author{
Shuangluo Xia, William H. Konigsberg*, and Jimin Wang* \\ Department of Molecular Biophysics and Biochemistry, Yale University, New Haven, Connecticut \\ 06520
}

\begin{abstract}
Results obtained using 2,4-difluorotoluene nucleobase (dF) as a non-polar thymine isostere by Kool and colleagues challenged the Watson-Crick dogma that hydrogen bonds between complementary bases are an absolute requirement for accurate DNA replication. Here we report crystal structure of an RB69 DNA polymerase L561A/S565G/Y567A triple mutant ternary complex with a templating dF opposite dTTP at $1.8 \AA$ A-resolution./ In this structure, direct hydrogen bonds were observed between: (i) dF and the incoming dTTP; (ii) dF and residue G568 of the polymerase and; (iii) $\mathrm{dF}$ and ordered water molecules surrounding the nascent base pair. Therefore, this structure provides evidence that a templating $\mathrm{dF}$ can form novel hydrogen bonds with the incoming dTTP and with the enzyme that differ from those formed with a templating dT.
\end{abstract}

\section{Keywords}

Difluorotoluene nucleotide; RB69 DNA polymerase; Hydrogen-bonding

\begin{abstract}
The 2'-deoxyribo-2,4-difluorotoluene nucleobase $\mathrm{dF}$ (Fig. 1), a reportedly non-polar, nonhydrogen-bonding isostere of $\mathrm{dT}^{1}$, can be recognized by both A and B family DNA polymerases (pols). ${ }^{2,3}$ When it is in the form of an incoming deoxynucleoside triphosphate, it is incorporated opposite a templating $\mathrm{dA}$ in preference to the other three naturally occurring dNTPs but with lower efficiency than dTTP. ${ }^{4}$ When $\mathrm{dF}$ is in the templating position, both A and B family DNA polymerases select dATP over the other dNTPs for primer-extension. ${ }^{5}$ We have determined the crystal structure of the ternary complex of a mutant RB69 DNA polymerase containing the primer/template duplex and the incoming dTTP opposite $\mathrm{dF}$ in the templating position at $1.8 \AA$ A-resolution to show that $\mathrm{dF}$ has a novel hydrogen bonding capability in the polymerase active site during DNA synthesis.
\end{abstract}

2,4-Difluorotoluene has been studied in solution when it partitions into the organic phase with a $\log \mathrm{P}$ value of 0.78 and it is indeed highly non-polar. ${ }^{3,6}$ When $\mathrm{dF}$ is part of a DNA duplex opposite dA, it destabilizes the DNA despite its shape similarity to $\mathrm{dT}^{7}{ }^{7}$ Therefore, it was thought that $\mathrm{dF}$ not only fails to hydrogen bond to $\mathrm{dA}$ in the complementary strand but actually results in a net repulsion between $\mathrm{dA}$ and $\mathrm{dF}$ because $\mathrm{dA}$ has a preference for hydrogen-bonding to water. ${ }^{8}$ When $\mathrm{dF}$ was placed in the middle of a Dickerson-Drew dodecamer DNA duplex opposite $\mathrm{dA}$, which was then bound to BhRNase $\mathrm{H}$, the $1.6 \AA$ resolution crystal structure of this complex showed that $\mathrm{dF}$ exhibited direct hydrogen bond

CORRESPONDING AUTHORS: jimin.wang@yale.edu, (203)-432-5737, william.konigsberg@yale.edu, (203)-785-5499.

Supporting Information Available: Experimental procedures. This material is available free of charge via the Internet at http://pubs.acs.org. 
(HB) engagement with $\mathrm{dA} .{ }^{9}$ However, such $\mathrm{dF} / \mathrm{dA}$ (or $\mathrm{rF} / \mathrm{rA}^{10}$ )-containing structures with $\mathrm{dF} / \mathrm{dA}$ embedded in the middle of duplexes, not at their ends, do not accurately reflect structural features of the nascent $\mathrm{dF} / \mathrm{dATP}$ base pair in a pol ternary complex during nucleic acid synthesis. Given what appears to be a conflicting interpretation about the ability of $\mathrm{dF}$ to participate in HB interactions, we decided to investigate its behavior when it was the templating partner in a nascent-base-pair binding pocket and a component of a ternary DNA pol:primer/template:dNTP complex. Since dF had not been studied in this context previously, we chose to determine crystal structures of a DNA polymerase ternary complex that met this requirement. This would provide the most direct evidence for its role during DNA synthesis. We designed a L561A/S565G/Y567A triple mutant RB69 pol that incorporated dAMP opposite $\mathrm{dF}$ more efficiently than the wild type (wt) enzyme and it accepted the other three dNTPs as part of the ternary complex where wt RB69pol did not. ${ }^{10}$ In addition the RB69pol triple mutant had the same pre-steady-state kinetic parameters for the incorporation of correct dNMPs as the wt RB69pol. ${ }^{10}$ Here we report the crystal structure of the RB69pol triple mutant ternary complex with dTTP opposite dF at $1.8 \AA$ resolution. The structure has been refined with a free R-factor of $20.3 \%$ (Table 1).

An overview of the ternary complex and a close-up of the electron density map for the nascent dTTP/dF base-pair are shown in Fig 2. The electron densities of the templating dF and the surrounding network of ordered water molecules were well-defined. In this structure, $\mathrm{dF}$ has a direct wobble HB between F4 of dF and N3-H of dTTP, and another HB between $\mathrm{F} 2$ of $\mathrm{dF}$ and the $\mathrm{C} \alpha-\mathrm{H}$ of G568. The HB distance between N3 of dTTP and F4 of $\mathrm{dF}$ is $2.97 \AA$, and the HB distance between $\mathrm{C} \alpha$ of G568 and F2 of dF is $2.96 \AA$ (Figure 2). The distance between the $\mathrm{C} 3-\mathrm{H}$ of dF and $\mathrm{O} 2$ of incoming dTTP is $3.7 \AA$, which is too great to form a $\mathrm{HB}$. In addition, the $\mathrm{C} 3-\mathrm{H}$ of $\mathrm{dF}$ was able to avoid contact with polar groups in such a way as to prevent ordered water molecules from being intercalated at the interface between dTTP and dF. We want to emphasize that the shape and hydrogen bond patterns observed here in the nascent dF/dTTP base pair differ from the dT/dT base pair embedded in the middle of a DNA duplex in complex with a polymerase, where two wobble inter-base hydrogen bonds are formed with inter-atomic distances of $2.91 \AA$ and $3.34 \AA$ for the O4/N3 and N3/O2 pairs, respectively. ${ }^{11}$

This structure provides direct evidence that a templating $\mathrm{dF}$ can form novel HBs that differ from those formed with a templating $\mathrm{dT}$. In the $\mathrm{dTTP} / \mathrm{dF}$ ternary complex, there are two direct HBs, one between $\mathrm{dF}$ and the incoming dTTP and the other between $\mathrm{dF}$ and the protein residue G568. In addition, F4 appears to HB to three ordered water molecules with HB distances varying from 2.90 to $3.34 \AA$. None of these features were anticipated since the chemical properties of $\mathrm{dF}$, determined in the absence of polymerase, indicated that it was non-polar and unable to participate in HB interactions. ${ }^{2,3}$ In addition, we also determined crystal structure of the RB69pol Y567A single mutant with a templating dF opposite dATP at slightly lower resolution $(2.3 \AA)$. As shown in supplementary fig 1 , the distance between F4 of dF and N6-H of incoming dATP is $4.3 \AA$, which is too great for a HB. Therefore, the direct $\mathrm{HB}$ between $\mathrm{dF}$ and incoming dTTP observed in the geometrically less-constrained triple mutant is not enforced by the enzyme, nor by the DNA backbone. However, the resolution of this $\mathrm{dF} / \mathrm{dATP}$-containing structure is not sufficiently high to observe all ordered water molecules near $\mathrm{F} 4$ of the templating $\mathrm{dF}$.

It is worth noting that the overall structure of the RB69 pol triple mutant ternary complex with dTTP opposite $\mathrm{dF}$ is almost superimposeable with that of the wt RB69 pol ternary complex with dATP opposite dT except for the nascent base-pair binding pocket, where the residues in the wt pol have been replaced by Ala or Gly. ${ }^{12}$ In addition, Gly568 has shifted down toward the interior of the polymerase allowing mispaired dNTPs to be accommodated in the ternary complex. Although the crystal structure reported here clearly demonstrates the 
partial polar character of a templating $\mathrm{dF}$, it does not provide an explanation for the base selectivity exhibited by $\mathrm{dF}$ for $\mathrm{dATP}$, an issue that will be addressed in a subsequent report. Data deposition: refined atomic coordinates and the observed structure factors have been deposited in the Protein Data Bank with PDB access code 3QEP and 3RWU.

\section{Supplementary Material}

Refer to Web version on PubMed Central for supplementary material.

\section{Acknowledgments}

We thank the staff of the NE-CAT beam-line 24-ID-C at the Advanced Photon Source of Argonne National Laboratory. This work was supported by NIH RO1-GM063276-09 (to W.H.K.) and by Steitz Center for Structural Biology, Gwangju Institute of Science and Technology (SCSB-GIST), Republic of Korea (to J.W.).

\section{References}

1. (a) Schweitzer BA, Kool ET. J Org Chem. 1994; 59:7238-7242. [PubMed: 20882116] (b) Schweitzer BA, Kool ET. J Am Chem Soc. 1995; 117:1863-1872. [PubMed: 20882111]

2. (a) Kool ET. Annu Rev Biochem. 2002; 71:191-219. [PubMed: 12045095] (b) Morales JC, Kool ET. Nat Struct Biol. 1998; 5:950-954. [PubMed: 9808038]

3. Kool ET, Sintim HO. Chem Comm. 2006:3665-3675. [PubMed: 17047807]

4. Moran S, Ren RX, Kool ET. Proc Natl Acad Sci. 1997; 94:10506-10511. [PubMed: 9380669]

5. (a) Moran S, Ren RX, Rumney S, Kool ET. J Am Chem Soc. 1997; 119:2056-2057. [PubMed: 20737028] (b) Lee H, Helquist SA, Kook ET, Johnson KA. J Biol Chem. 2008; 283:14402-14410. [PubMed: 17650502]

6. Barthel-Rosa L, Gladysz JA. Coord Chem Rev. 1999; 190-192:587-605.

7. (a) Guckian KM, Krugh TR, Kool ET. Nat Struct Biol. 1998; 5:954-959. [PubMed: 9808039] (b) Guckian KM, Schweitzer BA, Ren RX, Sheils CJ, Tahmassebi DC, Kool ET. J Am Chem Soc. 2000; 122:2213-2222. [PubMed: 20865137]

8. (a) Sharp KA, Nicholls A, Fine RF, Honig B. Science. 1991; 252:106-109. [PubMed: 2011744] (b) Zhang X, Lee I, Berdis AJ. Org Biomol Chem. 2004; 2:1703-1711. [PubMed: 15188037]

9. Pallan PS, Egli M. J Am Chem Soc. 2009; 131:12548-12549. [PubMed: 19685868]

10. Xia J, Noronha A, Toudjarska I, Li F, Akinc A, Braich R, Frank-Kamenetsky M, Rajeev KG, Egli M, Manoharan M. ACS Chem Biol. 2006; 1:176-183. [PubMed: 17163665]

11. Johnson SJ, Beese LS. Cell. 2004; 116:803-816. [PubMed: 15035983]

12. Zhang H, Beckman J, Wang J, Konigsberg WH. Biochemistry. 2009; 48:6940-6950. [PubMed: 19522539] 

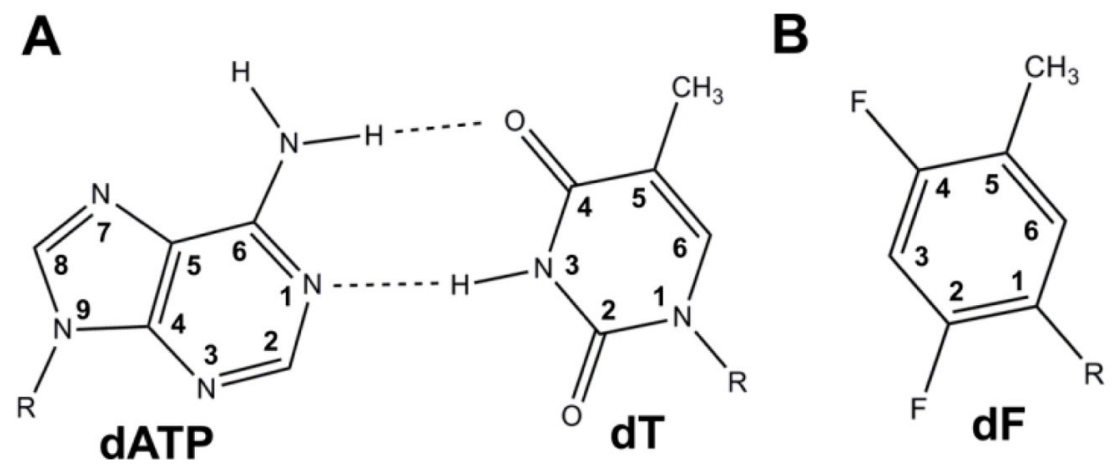

Figure 1.

An isostere of dT. (A) Complementary dA/dT base pair. (B). 2,4-Difluorotoluene analogue. 

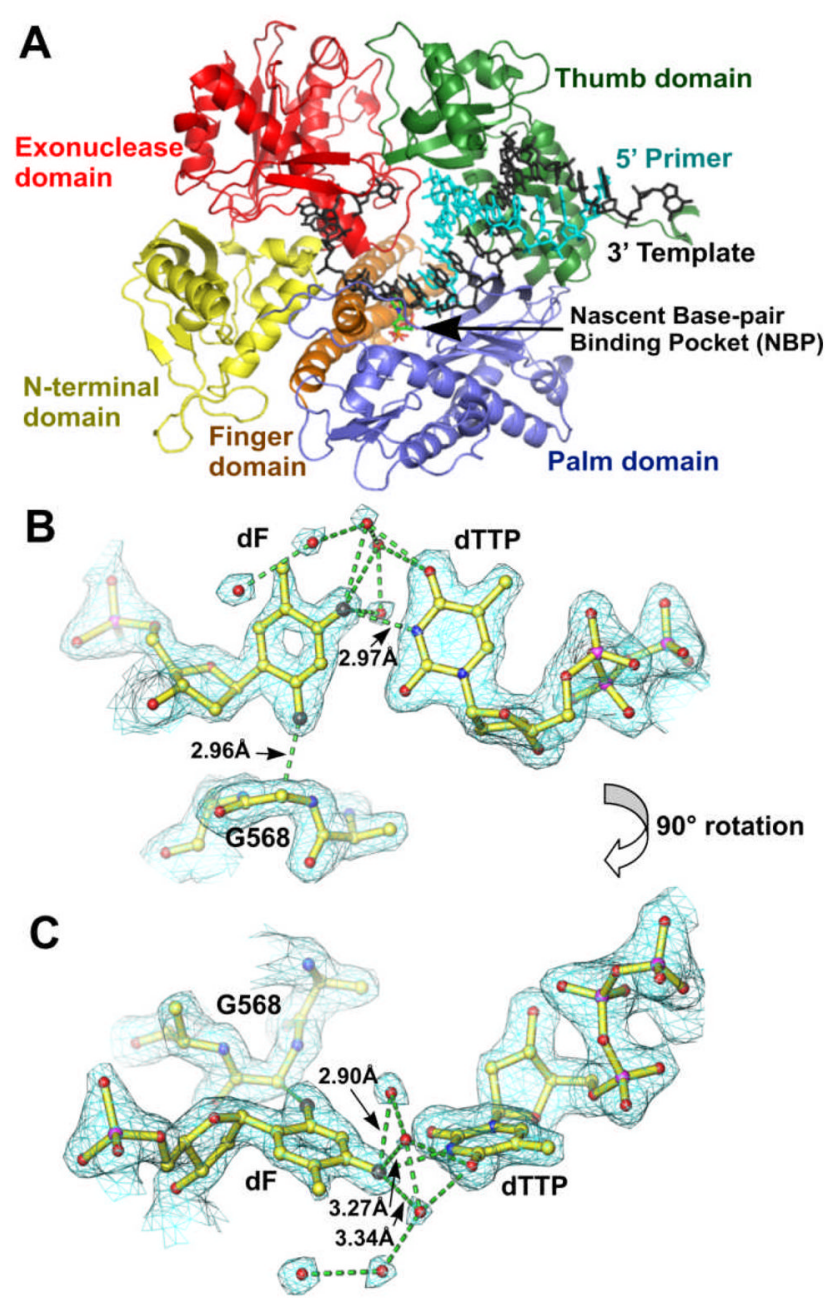

Figure 2.

Overall structure of the $\mathrm{dF} / \mathrm{dTTP}$-containing ternary complex of the RB69pol triple mutant. (A). The pol structure is colored by domains: the N-terminal domain (residues 1-108 and 340-382) in yellow, exonuclease (residues 109-339) in red, Palm (residues 383-468 and 573-729) in purple, Fingers (residues 469-572) in orange, and Thumb (residues 730-903) in green. The primer and template strand of the DNA are shown in a stick representation, with the template in black and the primer in cyan. (B). A close-up of the nascent base pair superimposed onto the final $2 \mathrm{Fo}-\mathrm{Fc}$ map at $1.8-\AA$ resolution contoured at $1.0 \sigma$ (cyan chicken wires). (C). An orthogonal view of panel B. 
Table 1

Crystallographic statistics for data collection and refinement of the templating base dF-containing ternary complex of the RB69pol triple mutant

\begin{tabular}{|c|c|}
\hline & dTTP opposite dF \\
\hline Space group & $\mathrm{P} 2_{1} 2_{1} 2_{1}$ \\
\hline Unit cell (a, b, c (̊)) & $75.13,119.77,130.48$ \\
\hline Resolution $(\AA)^{a}$ & $50.0-1.80(1.83-1.80)$ \\
\hline No of unique reflections & 101,984 \\
\hline Redundancy & $4.2(2.8)$ \\
\hline Completeness (\%) & $98.4(87.0)$ \\
\hline $\mathrm{R}_{\text {merge }}(\%)^{b}$ & $9.8(89.2)$ \\
\hline$I / \sigma$ & $14.0(1.3)$ \\
\hline \multicolumn{2}{|l|}{ Final model content: } \\
\hline Amino acid residues & 903 \\
\hline Water molecules & 1,234 \\
\hline $\mathrm{Ca}^{2+}$ ions & 4 \\
\hline Template nucleotides & 18 \\
\hline Primer nucleotides & 13 \\
\hline dTTP & 1 \\
\hline $\mathrm{R}(\%)^{c}$ & $16.7(29.2)$ \\
\hline $\mathrm{R}_{\text {free }}(\%)^{c}$ & $20.3(32.6)$ \\
\hline \multicolumn{2}{|l|}{ r.m.s.d. ${ }^{d}$} \\
\hline Bond length $(\AA)$ & 0.008 \\
\hline Bond angles $\left({ }^{\circ}\right)$ & 1.16 \\
\hline $\mathrm{R}_{\mathrm{o} 2 \mathrm{p}}^{e}$ & 2.73 \\
\hline PDB accession & 3QEP \\
\hline
\end{tabular}

${ }^{a}$ Statistics for the highest resolution shell are in parenthesis.

${ }^{b} \mathrm{R}_{\text {merge }}=\Sigma_{\mathrm{hkl}} \Sigma_{\mathrm{j}}\left|I_{\mathrm{j}}(\mathrm{hkl})-\left\langle\mathrm{I}_{\mathrm{j}}(\mathrm{hkl})\right\rangle\right| / \Sigma_{\mathrm{hkl}} \Sigma_{\mathrm{j}}\langle I(\mathrm{hkl})\rangle$, statistics for merging all observations for given reflections.

$c_{\mathrm{R}}=\Sigma_{\mathrm{hkl}}\left|\mathrm{F}_{\mathrm{Obs}}(\mathrm{hkl})-\mathrm{F}_{\mathrm{calc}}(\mathrm{hkl})\right| \Sigma_{\mathrm{hkl}} \mathrm{F}_{\mathrm{Obs}}(\mathrm{hkl})$, statistics for crystallographic agreement between the measured and model-calculated amplitudes.

$\mathrm{R}_{\text {free }}$ is the agreement for cross-validation data set.

$d_{\text {Root mean squares deviations (r.m.s.d.) to ideal values. }}$

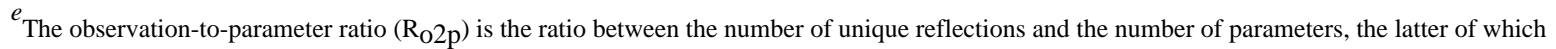
is four times the number of atoms (i.e, $x, y, z$, and $\mathrm{B}$ parameters). 